\title{
On a Class of Inclusions in Ordered Spaces
}

\author{
N. B. Huy, D. B. Dung and N. H. Khanh
}

\begin{abstract}
Let $W$ be a non-empty set, $X$ an ordered topological space, $L: W \rightarrow X$ a single-valued operator and $N: W \rightarrow 2^{X} \backslash\{\emptyset\}$ a set-valued operator. Under approximate assumptions on monotonicity of $L$ and $N$ we prove existence results for inclusions $L x \in N x$. An application of the obtained results to implicit elliptic equations of the form $L u=f(x, u, L u)$ is given.

Keywords: Increasing multi-valued operators, inclusions, ordered spaces, implicit elliptic equations
\end{abstract}

AMS subject classification: 35J65, 47H04, 47H07, 47H10

\section{Introduction}

Fixed point theorems for single-valued increasing operators in ordered spaces are widely investigated and have found various applications to differential equations (see $[3,6]$ and references therein). Recently, for some operators $L$ and $N$, the existence of solutions for operator equations of the type $L x=N x$ in ordered spaces with applications to implicit differential equations were given in [4]. In the present paper we shall deal with similar results for multi-valued operators.

The notion of monotonicity of multi-valued operators and the existence of fixed points for increasing multi-valued operators were first given by Nishnianidze in [11]. Since the appearance of that paper the study of increasing multi-valued operators has received little attention. In our recent paper [9] we have presented some simple fixed point theorems for increasing multi-valued operators. More interesting fixed point theorems with an application to discontinuous elliptic equations are given in [7,8]. Further extensions have been obtained in [5].

Nguyen Bich Huy and Dao Bao Dung: College of Education, Dept. Math., 280 An Duong Vuong, Ho Chi Minh City, Vietnam; nguyenbichhuy@hcm.vnn.vn

Nguyen Huu Khanh: College of Sciences, Dept. Math., Cantho Univ., 3-2 Street, Cantho Province, Vietnam

ISSN 0232-2064 / \$2.50 C Heldermann Verlag Berlin 
In the present paper we shall use a general fixed point theorem of [5] to prove existence results for inclusions of the form $L x \in N x$. Then we demonstrate applicability of the obtained results, using them as an alternative way to prove the existence for solutions of implicit elliptic equations.

\section{Solvability of Inclusions in ordered Spaces}

Let $(X, \leq)$ be an ordered topological space, that is a topological space $X$ in which there is defined an ordering " $\leq$ " such that the sets $\{y \in X \mid y \leq x\}$ and $\{y \in X \mid x \leq y\}$ are closed for all $x \in X$. Throughout this section we assume that $(X, \leq)$ has the following property:

(C) Each well-ordered chain $C$ of $X$ whose increasing sequences converge contains an increasing sequence which converges to sup $C$.

It is proved that each ordered metric space has the property (C) [6: Proposition 1.1.5] and that each ordered normed space equipped with weak topology has property $(\mathrm{C})$ [3: Lemma A.3.1].

Following Nishnianidze [11] we define a pre-ordering in the set $2^{X} \backslash\{\emptyset\}$ as

$$
A<B \text { if and only if } \forall a \in A \quad \exists b \in B: a \leq b \text {. }
$$

A set $A \subset X$ is said to be directed upwards if

$$
\forall x, y \in A \quad \exists z \in A: \quad x \leq z \text { and } y \leq z
$$

A multi-valued operator $F: M \subset X \rightarrow 2^{X} \backslash\{\emptyset\}$ is called increasing if $x, y \in M$ with $x \leq y$ implies $F x<F y$. If $x \in F x$, then $x$ is called fixed point of $F$.

In the sequence we need the following two general fixed point theorems.

Theorem A [5]. Let $F: M \subset X \rightarrow 2^{X} \backslash\{\emptyset\}$ satisfy the following hypotheses:

(F1) The set $M_{0}=\{x \in M \mid x<F x\}$ is non-empty.

(F2) If $\left\{x_{n}\right\}$ and $\left\{y_{n}\right\}$ are increasing sequences in $M_{0}$ and if $y_{n} \in F x_{n} \quad(n \in$ $\mathbb{N})$, then $\left\{y_{n}\right\}$ converges in $X$.

(F3) If $x \in M_{0}$ and $x \leq y \in F x$, then $y \in M_{0}$.

(F4) Each increasing and convergent sequence of $M_{0}$ has an upper bound in $M_{0}$.

Then the set $M_{0}$ has a maximal element, and each maximal element of $M_{0}$ is also a maximal fixed point of $F$. Further, if $M_{0}$ is directed upwards, then $\max M_{0}$ exists and is the greatest fixed point of $F$. 
Theorem B [6]. Let $X$ be an ordered metric space and $f: M \subset X \rightarrow M$ be a single-valued increasing operator such that:

1) There exists an element $u_{0} \in M$ satisfying $u_{0} \leq f\left(u_{0}\right)$.

2) The sequence $\left\{f\left(u_{n}\right)\right\}$ converges in $M$ whenever $\left\{u_{n}\right\}$ is an increasing sequence in $M_{0}=\left\{u \in M \mid u_{0} \leq u \leq f(u)\right\}$.

Then $f$ has a least fixed point in $M_{0}$.

Let $W$ be a non-empty set, $X$ an ordered topological space, $L: W \rightarrow X$ a single-valued operator and $N: W \rightarrow 2^{X} \backslash\{\emptyset\}$ set-valued. Motivated by the study in [4] we want to establish some existence results for solutions of the inclusion

$$
L u \in N u \text {. }
$$

By adaptation of a monotonicity condition in [4] to the multi-valued case we can apply Theorem A to obtain the solvability of inclusion (1).

Theorem 1. Let $L$ and $N$ be operators satisfying the following hypotheses:

(N1) The set $W_{0}=\{u \in W \mid L u<N u\}$ is non-empty.

(N2) If $u_{n} \in W_{0}$ and $y_{n} \in N u_{n}(n \in \mathbb{N})$ such that $\left\{L u_{n}\right\}$ and $\left\{y_{n}\right\}$ are increasing, then $\left\{y_{n}\right\}$ converges.

(N3) If $u \in W_{0}$ and $L u \leq x \in N u$, then $x \in L\left(W_{0}\right)$.

(N4) Every increasing and convergent sequence of $L\left(W_{0}\right)$ has an upper bound in $L\left(W_{0}\right)$.

Then inclusion (1) has a solution.

Proof. Let us define two multi-valued operators

$$
\begin{aligned}
L^{-1}: & L\left(W_{0}\right) \rightarrow 2^{W_{0}} \backslash\{\emptyset\} \\
F & : L\left(W_{0}\right) \rightarrow 2^{X} \backslash\{\emptyset\}
\end{aligned}
$$

by

$$
\begin{aligned}
L^{-1} x & =\left\{u \in W_{0} \mid L u=x\right\} \\
F x & =N \circ L^{-1} x=\bigcup_{u \in L^{-1} x} N u .
\end{aligned}
$$

Clearly, if $x$ is a fixed point of $F$ and $u \in L^{-1} x$, then $u$ will be a solution of inclusion (1). We shall verify that the defined above operator $F$ satisfies all hypotheses (F1) - (F4) of Theorem A.

First we observe from the definition of $F$ that

$$
y \in F x \quad \Longleftrightarrow \quad \exists u \in W_{0}: y \in N u \text { and } L u=x
$$


and, consequently,

$$
u_{0} \in W_{0} \quad \Longrightarrow \quad x_{0}=L u_{0} \in M_{0}=\{x \mid x<F x\}
$$

Assuming that $x \leq y \in F x$, we shall prove $y \in M_{0}$. By (2) there exists $u \in W_{0}$ such that $x=L u \leq y \in N u$ and so $y=L v$ for some $v \in W_{0}$ by Hypothesis (N3). Therefore, $y \in M_{0}$ by (3). If $\left\{x_{n}\right\}$ and $\left\{y_{n}\right\}$ are two increasing sequences of $M_{0}$ such that $y_{n} \in F x_{n}$, then there exists $u_{n} \in W_{0}$ satisfying $x_{n}=L u_{n}$ and $y_{n} \in N u_{n} \quad(n \in \mathbb{N})$ and hence $\left\{y_{n}\right\}$ converges by Hypothesis (N2). Thus, Hypothesis (F2) of Theorem A holds.

Finally, if $\left\{x_{n}\right\} \subset M_{0}$ is an increasing and convergent sequence, then $\left\{x_{n}\right\}$ has an upper bound in $M_{0}$. Indeed, we have $x_{n}=L u_{n} \quad(n \in \mathbb{N})$ with $\left\{u_{n}\right\} \subset W_{0}$ and hence there exists $u \in W_{0}$ such that $L u_{n} \leq L u$ by Hypothesis (N4) or, equivalently, $x_{n} \leq x$ with $x=L u \in M_{0}$. The proof is complete

Theorem 2. Let $N$ and $L$ be operators satisfying Hypotheses (N1), (N2), (N4) of Theorem 1 and the following hypothesis:

(N5) The operator $L$ is surjective, moreover $L u \leq L v$ implies $N u<N v$.

Then inclusion (1) has a solution.

Proof. It is sufficient to show that Hypothesis (N3) of Theorem 1 holds. Assume $L u \leq x \in N u$ and choose $v \in W$ such that $x=L v$. We have $L v<N u$ and $L u \leq L v$. This implies $N u<N v$ and so $L v<N v$. Thus $x \in L\left(W_{0}\right)$ and Hypothesis (N3) holds

Theorem 3. Let Hypotheses (N1) and (N2) of Theorem 1 and Hypothesis (N5) of Theorem 2 hold. Assume, in addition, the following:

(N6) For each $u \in W$ the set $N u$ is directed upwards and each increasing sequence of $N u$ converges to an element of $N u$.

Then inclusion (1) has a solution.

Proof. Let us prove that the combination of Hypotheses (N5) and (N6) implies Hypothesis (N4). For this assume that $\left\{L u_{n}\right\}$ is an increasing sequence in $L\left(W_{0}\right)$ such that $x=\lim L u_{n}$ exists in $X$. Assuming $x=L u$, we have from Hypothesis (N5) that $N u_{n}<N u$. Since $L u_{n}<N u_{n}$, then $L u_{n}<N u$ whence there is $y_{n} \in N u$ such that $L u_{n} \leq y_{n}(n \in \mathbb{N})$. Since $N u$ is directed upwards, we can assume that $\left\{y_{n}\right\}$ is increasing. Therefore, $y=\lim y_{n}$ exists and belongs to $N u$. Assuming $y=L v$ we have $L u \leq L v$, hence $N u<N v$. Since $L u<N u$ we have $L v<N v$ and so $L u_{n} \leq L v \in L\left(W_{0}\right)$. Thus, Hypothesis (N4) holds. The theorem is completely proved 


\section{Applications to implicit elliptic equations}

Let us consider the implicit elliptic boundary value problem

$$
\left.\begin{array}{rlrl}
L u & =f(x, u, L u) & & \text { in } \Omega \\
u=0 & & \text { on } \partial \Omega
\end{array}\right\}
$$

in a bounded domain $\Omega \subset \mathbb{R}^{N}(N \geq 3)$ with smooth boundary $\partial \Omega$, where $L$ is a second order elliptic operator in divergence form $L u=-\operatorname{div}(A(x) \cdot \nabla u)$ with $A(x)=\left(a_{i j}(x)\right)$ a symmetric matrix and $a_{i j} \in L^{\infty}(\Omega)$ satisfying for some positive number $\mu$

$$
A(x) \xi \xi \geq \mu|\xi|^{2} \quad \text { for a.e } x \in \Omega \text { and all } \xi \in \mathbb{R}^{N} .
$$

Problem (4) and its variants have been studied under various conditions on the function $f$ by Carl, Heikkilä, Marano and others (see [4, 8] and references therein). We shall consider problem (4) for two cases. In the first case we impose the same monotonicity condition on $f$ as in [4] but the growth conditions are different. In the second case the function $f=f(x, u, v)$ is assumed to be non-decreasing in $u$ and continous in $v$. To study the first case we apply Theorem 2, while the second case will be considered by Theorem 3 .

In this section we make blanked assumption that the function $f: \Omega \times$ $\mathbb{R}^{+} \times \mathbb{R} \rightarrow \mathbb{R}$ is sup-measurable. Moreover:

(H1) $0 \leq f(x, u, v) \leq a(x)|u|^{\alpha}+b(x)|v|^{\beta}$ for some $\alpha, \beta \in(0,1), a \in L^{p_{1}}(\Omega)$ and $b \in L^{p_{2}}(\Omega)$ where $p_{1}=\frac{2^{*}}{2^{*}-1-\alpha}, p_{2}=\frac{2^{*}}{\left(2^{*}-1\right)(1-\beta)}$ and $2^{*}=\frac{2 N}{N-2}$.

(H2) The function $u \mapsto f(x, u, v)$ is non-decreasing for all $v \in \mathbb{R}$ and a.e $x \in \Omega$.

(H3) There are a non-decreasing function $g: \mathbb{R} \rightarrow \mathbb{R}$ with $g(0)=0$ and an open bounded subset $\Omega^{\prime} \Subset \Omega$ satisfying $f(x, u, v) \geq g(u)$ for all $(x, u, v) \in \Omega^{\prime} \times \mathbb{R}^{+} \times \mathbb{R}$ and $\lim _{u \rightarrow 0^{+}} \frac{g(u)}{u}>\lambda_{1}$ where $\lambda_{1}$ is the first eigenvalue of the operator $L$ on $\Omega^{\prime}$.

Lemma. There exists a function $u_{0} \in W_{0}^{1,2}(\Omega)$ such that $L u_{0} \in L^{\left(2^{*}\right)^{\prime}}$ and

$$
L u_{0} \leq f\left(x, u_{0}, L u_{0}\right) \quad \text { a.e on } \Omega .
$$

Proof. Let $v_{0}=\varepsilon \varphi$, where $\varphi$ is the first eigenfunction of the operator $L$ on $\Omega^{\prime}$, set to $O$ in $\Omega \backslash \Omega^{\prime}$ and $\varepsilon>0$ is a number. Since $v_{0} \in L^{2^{*}}(\Omega)$ and $\left(2^{*}\right)^{\prime}<2^{*}$, we have $v_{0} \in L^{\left(2^{*}\right)^{\prime}} \subset W^{-1,2}$. Therefore, there exists $u_{0} \in W_{0}^{1,2}(\Omega)$ such that $L u_{0}=v_{0}$. It follows from results of [2] that $L v_{0} \leq \lambda_{1} v_{0}$ in the weak sense, consequently

$$
\left\langle L v_{0}-\lambda_{1} L u_{0}, w\right\rangle=\left\langle L v_{0}-\lambda_{1} v_{0}, w\right\rangle \leq 0
$$


for all $0 \leq w \in W_{0}^{1,2}(\Omega)$ where $\langle\cdot, \cdot\rangle$ stands for the dual pairing between $W_{0}^{1,2}(\Omega)$ and $W_{0}^{-1,2}(\Omega)$. Choosing $w=\left(v_{0}-\lambda_{1} u_{0}\right)^{+}$as a test function we conclude $v_{0} \leq \lambda_{1} u_{0}$ a.e on $\Omega$. Therefore, if $\varepsilon$ is small we have by condition (H3) that

$$
L u_{0}-f\left(x, u_{0}, L u_{0}\right) \leq \lambda_{1} u_{0}-g\left(u_{0}\right) \leq 0
$$

a.e on $\Omega$

Theorem 4. Let Hypotheses (H1) - (H3) hold. Assume, in addition, the following:

(H4) The function $v \mapsto f(x, u, v)$ is non-decreasing for all $u \in \mathbb{R}$ and a.e. $x \in \Omega$.

Then problem (4) has a non-trivial solution.

Proof. Let $u_{0}$ and $v_{0}$ be as in Lemma and $p=\left(2^{*}\right)^{\prime}=\frac{2^{*}}{2^{*}-1}$. We define the set

$$
W=\left\{u \in W_{0}^{1,2}(\Omega) \mid u \geq u_{0} \text { and } L u \in L^{p}(\Omega)\right\} .
$$

For every $u \in W$ let us consider the problem of finding $v \in L^{p}(\Omega)$ such that

$$
v(x)=f(x, u(x), v(x)):=F_{u}(v) \quad \text { for a.e } \quad x \in \Omega \text {. }
$$

The function $F_{u}(v)$ is measurable and satisfies

$$
\begin{aligned}
& \left(\int_{\Omega}\left|F_{u}(v)\right|^{p} d x\right)^{\frac{1}{p}} \\
& \quad \leq\left(\int_{\Omega} a^{p}(x)|u(x)|^{\alpha p} d x\right)^{\frac{1}{p}}+\left(\int_{\Omega} b^{p}(x)|v(x)|^{\beta p} d x\right)^{\frac{1}{p}} \\
& \quad \leq\|a\|_{p_{1}}\|u\|_{p^{\prime}}^{\alpha}+\|b\|_{p_{2}}\|v\|_{p}^{\beta} .
\end{aligned}
$$

Therefore, $F_{u}$ is a map from $L^{p}(\Omega)$ into itself, it is increasing and satisfies $F_{u}\left(v_{0}\right) \geq v_{0}$. Moreover, if $\left\{v_{n}\right\}$ is an increasing sequence such that $v_{n} \leq$ $F_{u}\left(v_{n}\right)$, then it follows from $(7)$ that the sequence $\left\{F_{u}\left(v_{n}\right)\right\}$ is bounded, hence being increasing it converges. Consequently, the solution set of equation (6), which we denote by $N u$, is non-empty by Theorem B. Problem (4) is now reduced to the inclusion $L u \in N u$, which will be solved by applying Theorem 2 for $W$ defined above and $X=L^{p}(\Omega)$.

Let us verify conditions (N1), (N2), (N4) and (N5). If $L u_{1} \leq L u_{2}$, then $u_{1} \leq u_{2}$ and we need to show $N u_{1}<N u_{2}$. For an element $v_{1} \in N u_{1}$ one has $v_{1}=F_{u_{1}}\left(v_{1}\right) \leq F_{u_{2}}\left(v_{1}\right)$. Therefore, equation (6) with $u=u_{2}$ has a solution 
$v \geq v_{1}$ by Theorem B. Thus, there exists $v \in N u_{2}$ such that $v \geq v_{1}$. It follows from the definition of $N u$ that

$$
v_{1}, v_{2} \in N u \quad \Longrightarrow \quad \max \left(v_{1}, v_{2}\right) \in N u .
$$

Hence, the set $N u$ is directed up-wards.

Considering increasing sequences $\left\{v_{n}\right\}$ and $\left\{L u_{n}\right\}$ satisfying $v_{n} \in N u_{n}$ and $L u_{n}<N u_{n}$, we shall show that $\left\{v_{n}\right\}$ converges. Actually, from property (8) we can construct a sequence $\left\{w_{n}\right\}$ such that $v_{n} \leq w_{n}$ and

$$
\begin{aligned}
w_{n} & =F_{u_{n}}\left(w_{n}\right) \\
L u_{n} & \leq w_{n} .
\end{aligned}
$$

Applying estimation (7) for $u=u_{n}$ and $v=w_{n}$ we get

$$
\left\|w_{n}\right\|_{p} \leq C\left(1+\left\|u_{n}\right\|_{p^{\prime}}^{\alpha}\right) .
$$

Using $u_{n}$ as a test function in (10), by the inequalities of Hölder and Sobolev we obtain

$$
C\left(\int_{\Omega} u_{n}^{2^{*}} d x\right)^{\frac{2}{2 *}} \leq \mu \int_{\Omega}\left|\nabla u_{n}\right|^{2} d x \leq\left(\int_{\Omega}\left|w_{n}\right|^{p} d x\right)^{\frac{1}{p}}\left(\int_{\Omega} u_{n}^{2^{*}} d x\right)^{\frac{1}{2 *}}
$$

which implies $\left\|u_{n}\right\|_{2^{*}} \leq \frac{1}{C}\left\|w_{n}\right\|_{p}$. This estimation and (11) prove the boundedness of $\left\{w_{n}\right\}$. The sequence $\left\{v_{n}\right\}$ is increasing and bounded, hence it converges.

Finally, we verify condition (N4). If a sequence $\left\{L u_{n}\right\}$ is increasing and satisfies $L u_{n}<N u_{n}$, then we can construct an increasing sequence $\left\{w_{n}\right\}$ satisfying (9) - (10). The sequences $\left\{L u_{n}\right\}$ and $\left\{w_{n}\right\}$ converge in $L^{p}(\Omega)$ to some functions $L u$ and $w$, respectively. Since $L u_{n} \leq L u$ one has $u_{n} \leq u$, hence $L u \leq w \leq f(x, u, w)$ by letting $n \rightarrow \infty$ in the inequality $L u_{n} \leq w_{n} \leq$ $f(x, u, w)$. Consequently, the set $N u$ contains an element $v \geq L u$ and so $L u<N u$. Thus, we have proved that $L u$ is an upper bound of $\left\{L u_{n}\right\}$ in $L\left(W_{0}\right)$. The theorem is completely proved

Theorem 5. Let hypotheses (H1) - (H3) be satisfied. Assume, in addition, the following:

(H5) The function $v \mapsto f(x, u, v)$ is continuous for all $u \in \mathbb{R}$ and a.e. $x \in \Omega$. Then problem (4) has a solution.

Proof. Let $u_{0}, v_{0}$ and $W, X$ be defined as in the proof of Theorem 4 , and let us define a multi-valued operator $N$ that assigns to each function $u \in W$ the set

$$
N u=\{v \mid v \text { is measurable and } v(x)=f(x, u(x), v(x)) \text { a.e on } \Omega\} .
$$


Then problem (4) is reduced to the inclusion $L u \in N u$, which will be solved by applying Theorem 3 .

First we need to prove $N u \neq \emptyset$ for every $u \in W$. In fact, since $v_{0}(x) \leq$ $f\left(x, u(x), v_{0}(x)\right)$ and $f(x, u(x), v) \leq v$ for sufficiently large $v$, Hypothesis (H5) yields that the set

$$
H(x)=\{v \in \mathbb{R} \mid f(x, u(x), v)-v=0\}
$$

is non-empty and closed. Moreover, the multi-valued function $x \mapsto H(x)$ is measurable by [1: Theorem 8.2.9]. This implies $N u \neq \emptyset$ by [1: Measurable Selection Theorem 8.1.3]. Furthermore, it follows from Hypotheses (H1) and (H5) and the Dominated Convergence Theorem that the set $N u$ is closed in $L^{p}(\Omega)$ and condition (N6) holds. Clearly, property (8) also holds for the defined operator $N$. Since $L u_{1} \leq L u_{2}$ implies $u_{1} \leq u_{2}$, hence to verify condition (N5) it is sufficient to show $N u_{1}<N u_{2}$ if $u_{1} \leq u_{2}$. Indeed, for a given function $y \in N u_{1}$ we define a multi-valued function $x \mapsto G(x)$ by

$$
G(x)=\left\{v \in \mathbb{R} \mid y(x) \leq v=f\left(x, u_{2}(x), v\right)\right\}
$$

Since $y(x)=f\left(x, u_{1}(x), y(x)\right) \leq f\left(x, u_{2}(x), y(x)\right)$, hence the equation $v=$ $f\left(x, u_{2}(x), v\right)$ has a solution $v \geq y(x)$, so $G(x) \neq \emptyset$. Again, the Measurable Selection Theorem yields that the set $N u_{2}$ contains an element that is greater than $y$.

Condition N2 can be verified as in proof of Theorem 4. The proof is complete

Acknowledgements. The authors would like to express their thanks to the referees for helpful suggestions to improve the paper.

\section{References}

[1] Aubin, J.-P. and H. Frankowska: Set-Valued Analysis. Basel: Birkhäuser 1990.

[2] Boccardo, L. and L. Orsina: Sublinear equation in $L^{s}$. Houston J. Math. 1 (1994), 99 - 114.

[3] Carl, S. and S. Heikkilä: Differential Equations in Ordered Spaces. Boca Raton: Chapman \& Hall/CRC Press 2000.

[4] Carl, S. and S. Heikkilä: Operator and differential equations in ordered spaces. J. Math. Anal. Appl. 234 (1999), 31 - 54.

[5] Carl, S. and S. Heikkilä: Fixed point theorems for multivalued operators and applications to discontinuous quasilinear BVP's. Applicable Anal. (to appear). 
[6] Heikkilä, S. and V. Lakshmikantham: Monotone Iterative Techniques for Discontinuous Nonlinear Differential Equations. New York - Basel: Dekker 1994.

[7] Huy, N. B.: On a class of inclusions in ordered spaces. J. Sci. HCM City Coll. Edu. 26 (2001), $47-50$.

[8] Huy, N. B.: Fixed points of increasing multivalued operators and an application to quasimonotone elliptic equations. Nonlin. Anal. 51 (2002), $673-678$.

[9] Huy, N. B. and N. H. Khanh: Fixed point for multivalued increasing operators. J. Math Anal. Appl. 250 (2000), 368 - 371.

[10] Marano, S.: Implicit elliptic boundary value problems with discontinuous nonlinearities. Set-Valued Anal. 4 (1996), 287 - 300.

[11] Nishnianidze, G.: Fixed points of monotonic multiple-valued operators. Bull. Aacd. Sci. Georgian SSR 114 (1984), 498 - 491.

Received 12.11.2002; in revised form 16.06.2003 